\title{
DOA-ALOHA: Slotted ALOHA for Ad Hoc Networking Using Smart Antennas
}

\author{
Harkirat Singh and Suresh Singh, Department of Computer Science \\ Portland State University, Portland, OR 97201 \\ \{harkirat,singh\}@cs.pdx.edu
}

\begin{abstract}
This paper develops a novel slotted ALOHA protocol (Direction-Of-Arrival ALOHA) for use in ad hoc networks where nodes are equipped with smart antennas. The protocol relies on the ability of the antenna and DOA algorithms to identify the direction of the desired signal and the direction of the interferers to maximize SINR (Signal to Interference and Noise Ratio) at the receiver. The performance of the protocol is evaluated using joint simulation in OPNET and Matlab. We compare the performance of our new protocol against recent directional MAC (Medium Access Control)[3] protocol. We show that DOA-ALOHA achieves significantly higher throughput than [3] despite its simplicity. The impact of using different number of antenna elements is also studied for this environment.
\end{abstract}

\section{INTRODUCTION}

Recently, there has been increasing interest in developing MAC protocols for use in ad hoc networks where nodes are equipped with directional antennas. Antenna models used include sectored fixed beam antennas, idealized adaptive array antennas, and steerable directional antennas. As previous researchers have shown, using directional antennas increases throughput because of better spatial reuse of the spectrum (see [10], [1], [3], [6]). However, we note that these previous works have not fully exploited the benefits of adaptive array antennas (or smart antennas) such as the ability to form nulls in the direction of interferers (resulting in high SINR) and the ability to determine the direction of transmitters (Direction of Arrival). We show that by exploiting these capabilities of smart antennas, a simple protocol can yield throughputs that are $2 \mathrm{x}-4 \mathrm{x}$ higher than one of the recent protocols [3]. We also note that our simulations use realistic antenna models unlike the idealized models used in many (with the exception of [6]) papers and, despite this, our protocol out performs most of these existing protocols.

The key problem in exploiting the capabilities provided by a smart antenna at a receiver is in determining the direction of the interfering signals (so as to place nulls in those directions) and forming a beam towards the transmitter. In order to do this, we have developed a modified version of the slotted ALOHA protocol in which a small initial portion of the slot is used for finding the direction of various transmitters. This is done by requiring each transmitter to transmit a pure tone towards its intended receiver for a short interval prior to transmitting the packet. The receiver runs a DOA (Direction Of Arrival) algorithm which provides information

This work is funded by the NSF under grant ANIR-0125728. about the received signal strength and direction of the different transmitters. This information is then used at each receiver to guide beamforming (beam and nulls) for the remaining duration of the slot. Upon correct packet reception, a receiver sends an ACK using the already formed beams.

We implemented smart antenna in Matlab and interfaced it with the physical layer of OPNET. In our study we used realistic antenna patterns with the side lobes. We carried out extensive simulations and obtain very high throughput in the single as well as multi-hop case. The remainder of the paper is organized as follows: in the next section we describe our system model. Section III describes the context for this work in relation to other research. Section IV describe our protocol DOA-ALOHA in more detail. Section V summarizes key results.

\section{SySTEM MODEL}

We assume that each node is equipped with a smart antenna system which is composed of a linear array of $M$ elements. For simplicity we assume that the antenna array is perpendicular to the $x-y$ plane in which the nodes lie. The reason for this assumption is that the beam formed by the antenna is symmetric about the antenna axis and is thus independent of the direction in which a node is "facing".

Figure 1 provides a schematic of an adaptive array smart antenna system. As illustrated in the figure, the antenna elements are separated from each other by a known distance $d$. We assume that the transmitter is located far enough away from the receiver that all the signals $S_{i}(t)$ arriving at the different antenna elements are parallel. However, since the elements are separated by distance $d$, the phase of the different signals is different. Let $w_{i}$ denote the phase and gain that is added to each signal $S_{i}(t)$. Then $z(t)$, the output sent to the receiver, can be written as,

$z(t)=\sum_{i=1}^{M} w_{i} S_{i}(t)+N(t)=A S(t) \sum_{i=1}^{M} w_{i} e^{-j \beta(i-1) d \cos \theta}+N(t)$

where $\beta=2 \pi / \lambda$ is the phase propagation factor, $\lambda$ is the wavelength, $A$ is an arbitrary gain constant, and $N(t)$ is AWGN noise. The weights $w_{i}$ used in this paper only shift the phase of the signal and leave the amplitude untouched.

\footnotetext{
${ }^{1}$ If we place the antenna array in the $x-y$ plane then the beam pattern formed in some direction will depend on the relative alignment of the antenna with respect to the $\mathrm{x}$ and $\mathrm{y}$ axes. This makes the analysis far more difficult without gaining any additional generality.
} 
If the desired direction is $\theta_{0}$, then the representation for the weights is,

$$
w_{i}=e^{j \beta(i-1) d \sin \theta_{0}}
$$

For a more comprehensive discussion, please see [5]. In Figure 2 we show the different antenna patterns formed by a linear array of $M=8,16$ elements when $\theta_{0}=45^{\circ}$. We note that as the number of elements increases, the beamwidth becomes narrower and directivity of the antenna increases. Further, we note that, rather than one beam, using a linear array results in two beams that can lead to greater interference. As we noted in the introduction, another beneficial feature of smart antennas is the ability of these antennas to form nulls in given directions. In fact, given $M$ elements, an antenna can form upto $M-1$ nulls. However, the shape of the desired beam can change depending on the number of and the direction of the nulls. In this work we use the MMSE (Minimum Mean Square Error) algorithm to determine weights $w_{i}$ to form nulls appropriately [5]. Figure 3 illustrates two cases when using $M=8$ antenna elements with $\theta=45^{\circ}$ being the desired direction. In the first case, we are forming only two nulls whereas in the second case we are forming six nulls. As can be seen, the shape of the beam and side lobes changes.

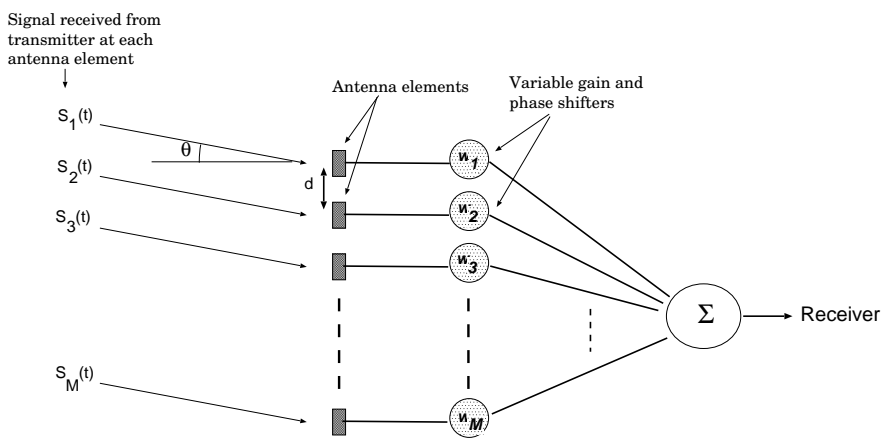

Fig. 1. Schematic of a smart antenna (adaptive linear array).

\section{RELATED WORK}

Recently there have been several papers that have looked at the problem of MAC design for ad hoc networks where nodes are equipped with directional antennas. The directional antenna models used include switched beam antennas (the antenna is sectored and one of these sectors is used depending the direction of the communicating node), multi-beam antennas (here more than one beam can be used simultaneously), and adaptive antenna arrays (here the beam can be made to point in any direction as described in section II).

[2] develops slotted scheduling-based MAC protocols for nodes equipped with directional antennas. The directional antenna considered is a multi-beam adaptive array antenna (MBAA) which is capable of forming multiple beams. The protocols assume that nodes can engage in several simultaneous transmissions. The key contribution of the paper is the development of a neighbor tracking scheme that is then used to schedule transmissions by each node in a distributed way.

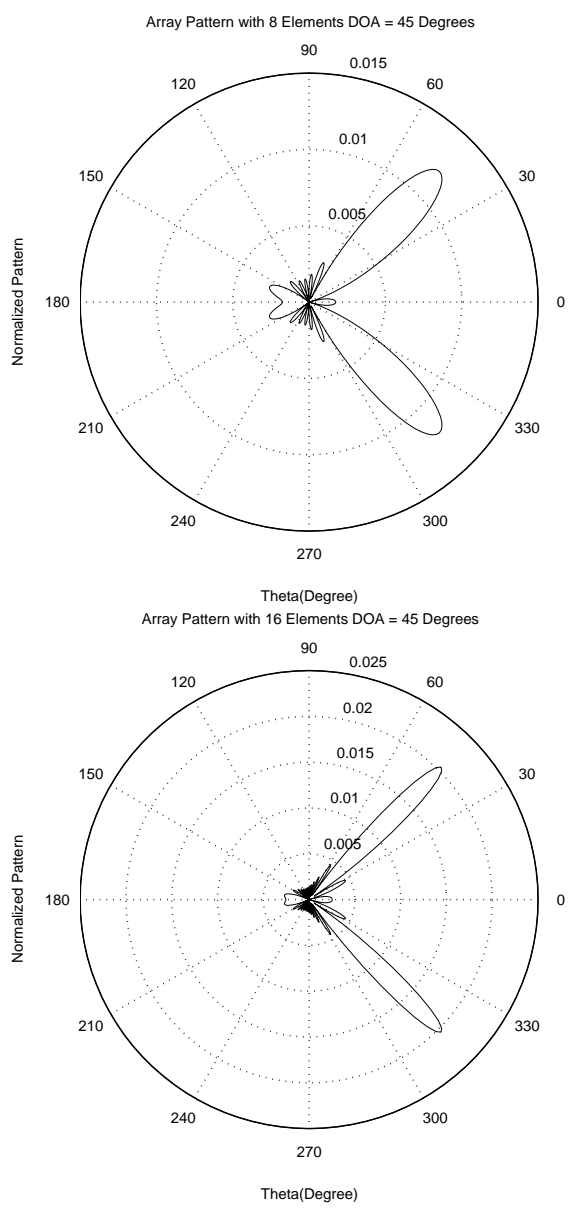

Fig. 2. Antenna patterns for 8 and 16 antenna elements.

[11] also develops MAC protocols for this scenario. They assume that the gain of the directional antenna equals omni directional antenna. An antenna is comprised of 4 antenna elements which can transmit in $90 \mathrm{deg}$ sectors only. Nodes are dependent on GPS or some other device to have position information of the sender and receiver. They present a MAC protocol based on Directional RTS/CTS or a combination of DRTS/O-CTS (Omnidirectional CTS). The protocol assumes that if a transmission is happening in some direction then it will defer all transmissions in that direction. Similarly, [4], [12] consider a switched beam antenna model with no nulling or DOA information. They use a directional antenna at the receiver. A second protocol based on DRTS/DCTS assumes two separate channels: one for data and another for signaling. These papers do not consider nulling or narrower beamwidths.

In [3] a novel multi-hop RTS is proposed to establish links between distant nodes and $G_{d}$ (directional gain) is assumed to be higher than $G_{0}$ (omni directional gain). The direction in which the main lobe is to be oriented is determined by the MAC protocol (which in turn is provided this information by the network layer which is assumed to be neighbor-aware). The authors note that node alignment negates the benefits achieved due to directional antennas, however, unaligned 

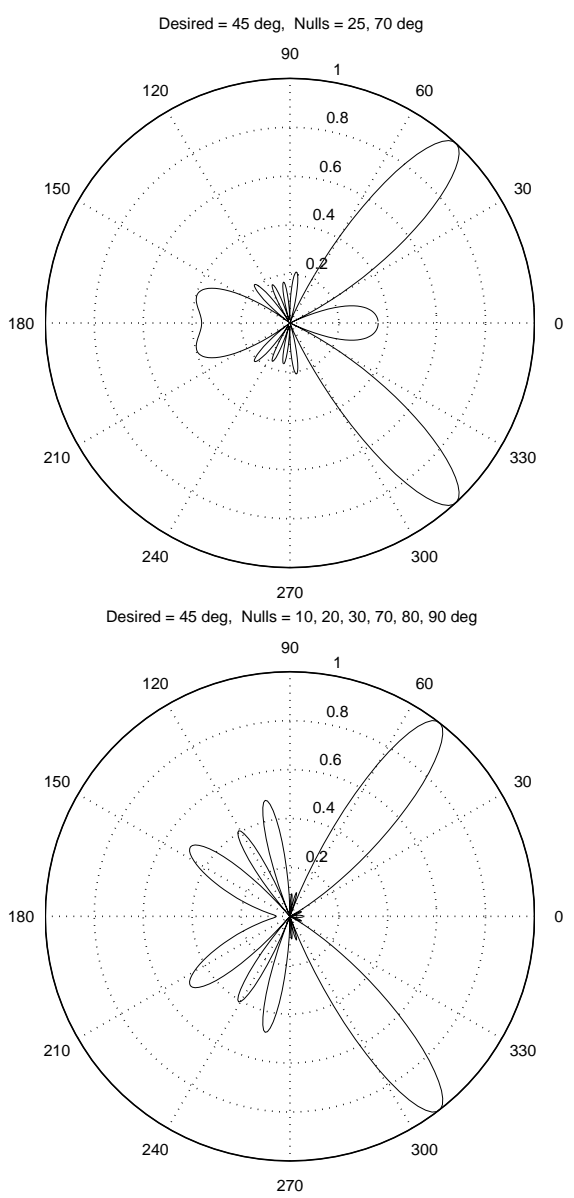

Fig. 3. Patters with 8 antenna elements and 2 or 6 nulls

routes enhances the spatial reuse. They show that their protocol has a 4-5x throughput as compared with 802.11.

In [8], [7] the authors assume that each node maintains neighbor Angle-SINR table (AST) and they provide a link state based table-driven routing and MAC protocol. Based on AST a node calculates an affinity for an angle which provides maximum SINR. Based on this a NLS Table is formed. Nodes in the beamformed region remain in the omni mode but they make nulls in the direction of ongoing transmissions.

[9] uses directional transmissions for control and data packets. It uses a directional-NAV table for transmission scheduling and collision avoidance. However, they do not exploit the capabilities of the smart antennas, such as beam steering and the placement of nulls in the direction of interferers.

In [6] a node caches AOA information based on signals received and nodes remain in promiscuous mode to cache signals. 802.11 specifications say that RTS needs to be transmitted 7 times, so a node will transmit 4 directional RTS and remaining the 3 as omni-directional RTS if there is no response to the directional RTS. A circular antenna with 6 elements is assumed, and a node is capable of electronically steering the boresight towards a specific direction. A constant beamwidth of $45 \mathrm{deg}$ assumed. However, it was observed that as the boresight changes, the side lobe pattern changes drastically.
The lesson is that the effects of side and back lobes cannot be ignored in the evaluation of network performance with directional antennas. [6] shows that using an ideal antenna results in a maximum throughput of $2.2 \mathrm{Mbps}$ while using a realistic antenna has a maximum throughput of only $1.4 \mathrm{Mbps}$. This fact, that antenna patterns matter in evaluating MAC behavior, is one that has largely been ignored by a great many authors. Following the lesson of [6], we use realistic antenna patterns in our studies.

\section{PROTOCOL DESCRIPTION: DOA-ALOHA}

In this section we describe the behavior of our protocol. However, before doing this, we need to make the following assumptions: (1) Nodes are aware of the angular location of each of their neighbors (as in [3]) since this information is needed at transmitters to form directed beams towards receivers; (2) For simplicity, we assume that all nodes use the same constant transmit power.

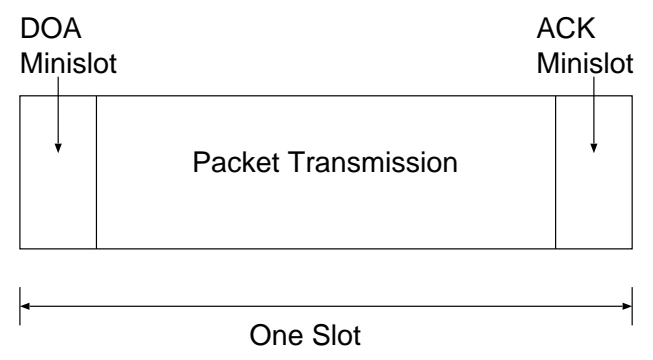

Fig. 4. Structure of a slot in DOA-ALOHA.

Figure 4 shows the form of the slots used in DOA-ALOHA and as shown, each slot is broken into three minislots. Our algorithm works as follows:

1) The first minislot in a slot is called the DOA-minislot and it is here that a node identifies the angular direction of all transmitters that it can hear. All transmitters transmit a simple tone (i.e., a sine wave) during the DOAminislot towards their intended receivers. The signal received at some receiver is thus the complex sum of all of these tones. The receiver runs a DOA algorithm (such as MUSIC [5]) to determine the angular direction of each of the transmitters and the received power from each transmitter.

2) Once a receiver determines the DOA of all transmitters it can hear, it forms its directed beam towards the one that has the maximum power and forms nulls in all the other identified directions.

3) The second (and largest) minislot is the packet transmission slot and it is here that the packets are transmitted. After the receiver has formed its beam and nulls as described above, it receives the packet from the transmitter. After receiving the packet, it looks at the header and rejects the packet if it was not the intended destination.

4) The last minislot is the ACK slot where the receiver transmits an ACK using the already formed beam to the sender (if the packet was not rejected and correctly 
received). When a transmitter does not receive an ACK, it retransmits the packet at a later time (this is exactly as in ALOHA).

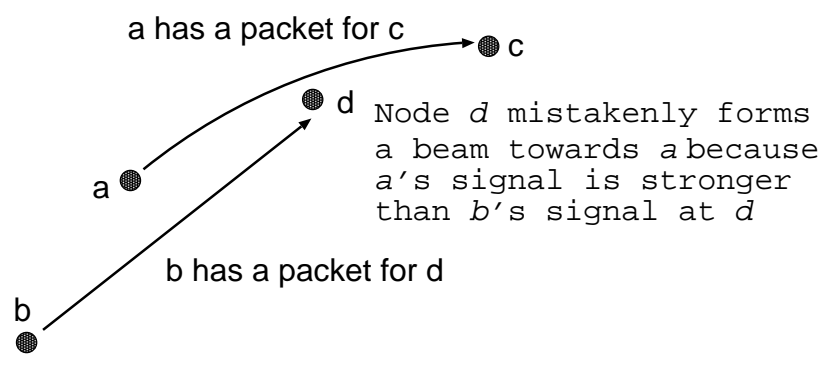

Fig. 5. False beamforming.

The intuition behind the receiver beamforming in the direction of the maximum signal is that, because of the directivity of the antenna, there is a high probability that it is the intended recipient for the packet. However, we note that in cases, as in Figure 5, the receiver $d$ incorrectly beamforms towards $a$ because $a$ 's signal is stronger than $b$ 's. While this is not a serious problem in most cases, we can envision scenarios where the $b \longrightarrow d$ transmission gets starved due to a large volume of $a \longrightarrow c$ traffic. An optimization we have therefore implemented is a single-entry cache scheme which works as follows:

- If a node beamforms incorrectly in a given timeslot, it remembers that direction in a single-entry cache.

- In the next slot, if the maximum signal strength is again in the direction recorded in the single-entry cache, then the node ignores that direction and beamforms towards the second strongest signal.

- If the node receives a packet correctly (i.e., it was the intended recipient), it does not change the cache.

- If it receives a packet incorrectly, it updates the cache with this new direction.

- If there is no packet in a slot from the direction recorded in the cache, the cache is reset.

This simple mechanism ensures that in cases similar to Figure 5, connections are not starved. However, we can construct more complex scenarios where a single-entry cache will fail to prevent starvation. In these cases, more sophisticated multipleentry caching schemes are required. However, in our simulations, we only use the single-entry caching scheme because the probability of more complex scenarios resulting in starvation are very rare.

\section{RESUlTS}

OPNET provides an excellent physical layer model but has a drawback in that it has a very idealistic directional antenna model. To overcome this drawback we implemented the smart antenna model (for a linear array of antenna elements) in Matlab and interfaced it with the physical layer of OPNET. We invoke Matlab to compute $w_{i}$ 's (section II) based on actual received signal strength $s_{i}(t)$ at each antenna element
TABLE I

OPNET SIMULATION PARAMETERS.

\begin{tabular}{|l|l|}
\hline \multicolumn{2}{|c|}{ Simulation Parameters } \\
\hline Background Noise + ambient Noise & $-143 \mathrm{~dB}$ \\
Propagation model & Free space \\
Bandwidth & $1,000 \mathrm{kHz}$ \\
Min frequency & $2,402 \mathrm{MHz}$ \\
Data Rate & $2000 \mathrm{kbps}$ \\
Carrier Sensing Threshold & $+3 \mathrm{~dB}$ \\
Minimum SINR & $9 \mathrm{~dB}$ \\
Bit Error & Based on BPSK \\
& Modulation curve \\
Maximum radio range & $250 \mathrm{~m}$ \\
\hline
\end{tabular}

as obtained from OPNET. We also modified OPNET's radio pipeline stage with the simulation parameters displayed in Table I.

We evaluate the performance of DOA-ALOHA using $5 \times 5$ mesh (as used in [3]) with four pre-defined flows. Figure 6 shows the network topology and flows used for two of these experiments. For the third experiment, we used a random node placement on the grid where a node's position is randomly shifted in the $\mathrm{x}$-axis and $\mathrm{y}$-axis by adding a displacement randomly selected from $[-150 \mathrm{~m},+150 \mathrm{~m}]$ and the flows are as in Figure 6(b). The traffic is CBR (Constant Bit Rate) which increases (per flow) from $75 \mathrm{kbps}$ to $2 \mathrm{Mbps}$. The packet size is 512 bytes. Figure 7 plots the aggregate throughput as a function of the data rate of one flow (for Figure 6(a)) for two antenna systems - one with 8 elements and one with 16 elements. Figure 8 does the same for Figure 6(b) and Figure 9 corresponds to the random mesh topology case. We used 10 different cases for random flows (Figure 6(b)) and randomly selected nodes. In order to make the comparison as fair as possible, we used the exact same parameters in our experiments as those described in [3].

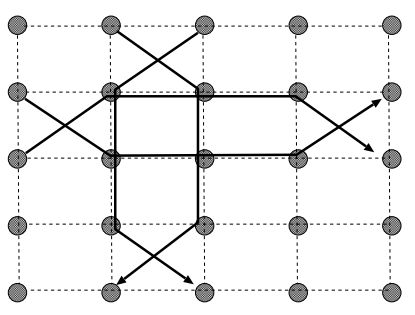

(a) Four flows (some alignment)

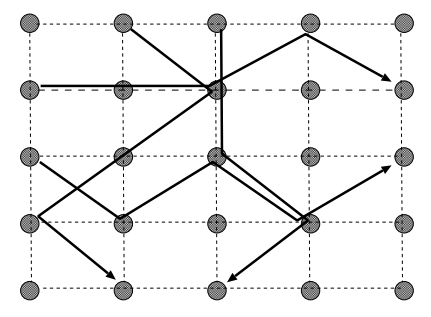

(b) Randomly selected flows
Fig. 6. $5 \times 5$ grid topology used to compare performance with [3].

We observe that using 16 antenna elements as opposed to 8 elements makes a big difference in aggregate throughput. This is because the beamwidth when using 16 elements is smaller than when using 8 elements which results in more simultaneous transmissions/slot. For the flows in Figure 6(a), (when flows are aligned), we did not notice much difference in the performance of 16 and 8 antenna elements but for Figure 6(b) and for random topologies we do see a significant difference. The reason is that when flows are not aligned, there is a greater potential for spatial reuse with 16 antenna elements (due to its smaller beamwidth). We note that the 
TABLE II

\begin{tabular}{|l|l|l|l|l|l|l|}
\hline & \multicolumn{2}{|c|}{ Mesh Figure 6(a) } & \multicolumn{2}{c|}{ Mesh Figure 6(b) } & \multicolumn{2}{c|}{ Random Mesh } \\
\hline & $\begin{array}{l}16 \text { Elements } \\
\left(\sim 40^{0}\right)\end{array}$ & $\begin{array}{l}\text { 8 Elements } \\
\left(\sim 70^{0}\right)\end{array}$ & $\begin{array}{l}\text { 16 Elements } \\
\left(\sim 40^{0}\right)\end{array}$ & $\begin{array}{l}\text { 8 Elements } \\
\left(\sim 70^{0}\right)\end{array}$ & $\begin{array}{l}16 \text { Elements } \\
\left(\sim 40^{0}\right)\end{array}$ & $\begin{array}{l}\text { 8 Elements } \\
\left(\sim 70^{0}\right)\end{array}$ \\
\hline DOA-ALOHA & $2500 \mathrm{kbps}$ & 2150 & 3000 & 2000 & 4250 & 3750 \\
\hline$[3]\left(45^{\circ}\right)$ & \multicolumn{3}{|c|}{$800 \mathrm{kbps}$} & \multicolumn{2}{c|}{1000} & \multicolumn{2}{c|}{1000} \\
\hline
\end{tabular}

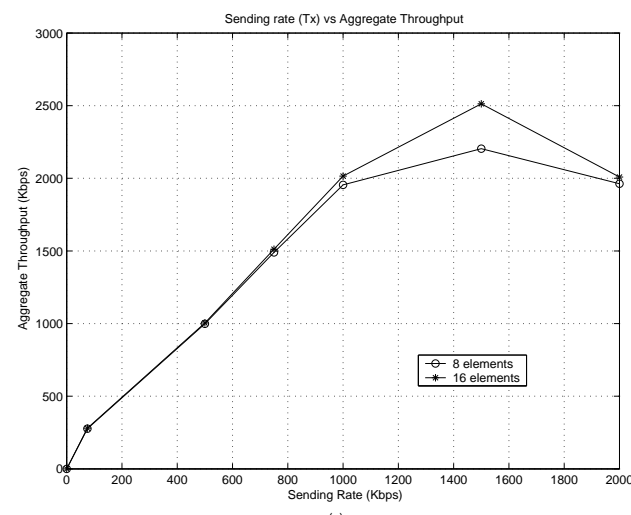

Fig. 7. Performance of our protocol in 6(a)

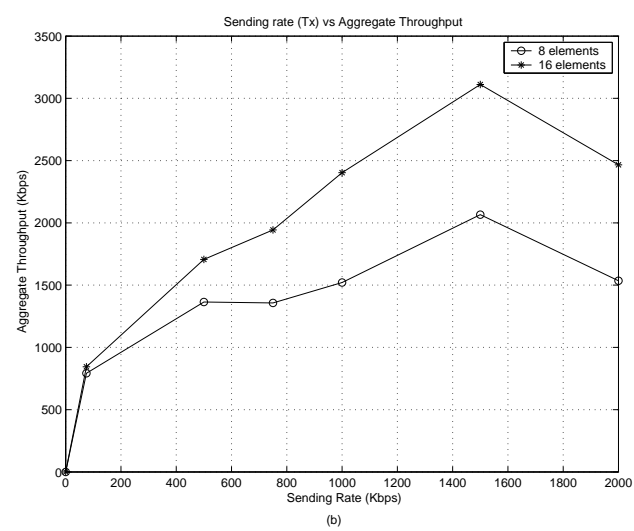

Fig. 8. Performance of our protocol in 6(b)

beamwidth used in [3] is $45^{\circ}$. In our case, the linear array creates two symmetric beams and we define beamwidth for our protocol as the sum of these two beams. For 16 antenna elements, we noted an average beamwidth of $20^{\circ}$ for each of the two symmetric beams formed with a linear array thus giving us an effective beamwidth of $40^{\circ}$ (adding the two beams). The effective beamwidth when using 8 antenna elements is approximately $70^{\circ}$. Table II summarizes our results and compares them with [3]. We observe that our protocol is $2 x-3 x$ better when we use 8 elements and is much better $(3 \mathrm{x}-4 \mathrm{x})$ for 16 elements.

\section{CONCLUSION}

In this paper we have presented DOA-ALOHA, a version of slotted ALOHA that uses DOA information at the receiver to beamform in a way that maximizes SINR. We compare the performance of our protocol against [3] and show that our protocol has a throughput of $2 \mathrm{x}-4 \mathrm{x}$ higher than the [3]. In

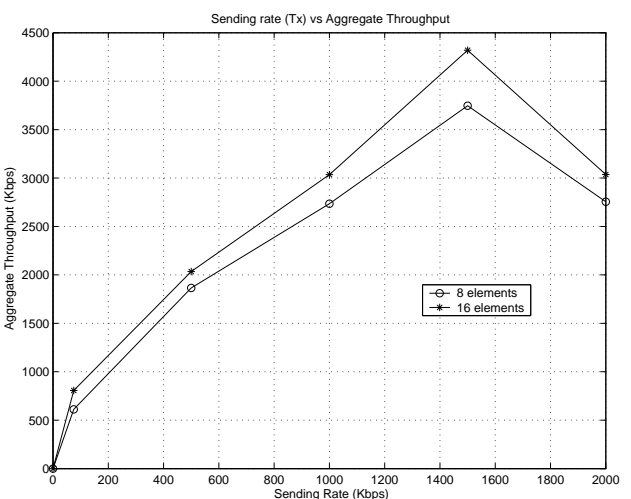

Fig. 9. Performance of our protocol in random grid topologies.

the future work we will examine the performance of 802.11 when using smart antennas.

\section{ACKNOWLEDGEMENTS}

We would like to thank OPNET for their technical support.

\section{REFERENCES}

[1] J. You A. Nasipuri, S. Ye and R. Hiromoto. A mac protocol for mobile ad hoc networks using directional antennas. In IEEE WCNC, 2000.

[2] Lichun Bao and J.J. Garcia-Luna-Aceves. Transmission scheduling in ad hoc networks with directional antennas. In ACM/SIGMOBILE MobiCom 2002, 23 - 28 Sep 2002.

[3] Romit Roy Choudhury, Xue Yang, Ram Ramanathan, and Nitin H. Vaidya. Using directional antennas for medium access control in ad hoc networks. In ACM/SIGMOBILE MobiCom 2002, 23 - 28 Sep 2002.

[4] Zhuochuan Huang and Chien-Chung Shen. A comparison study of omnidirectional and directional mac protocols for ad hoc networks. In IEEE Globecom 2002, 2002.

[5] J. C. Liberti and T. S. Rappaport. Smart Antennas for Wireless Communications. Prentice Hall, 1999.

[6] Rajiv Bagrodia Mineo Takai, Jay Martin and Aifeng Ren. Directional virtual carrier sensing for directional antennas in mobile ad hoc networks. In ACM/SIGMOBILE MobiHoc 2002, Oct 2002.

[7] S. Roy, D. Saha, S. Bandyopadhyay, T. Ueda, and S. Tanaka. A networkaware mac and routing protocol for effective load balancing in ad hoc wireless networks with directional antenna. In ACM Mobihoc'03, 1-3 June 2003.

[8] S. Horisawa S. Bandyopadhyay, K. Hausike and S. Tawara. An adaptive mac and directional routing protocol for ad hoc wireless networks using espar antenna. In ACM/SIGMOBILE MobiHoc 2001, Oct 2001.

[9] Gentian Jakllari Thanasis Korakis and Leandros Tassiulas. A mac protocol for full exploitation of directional antennas in ad-hoc wireless networks. In ACM Mobihoc'03, 1 - 3 June 2003.

[10] James Ward and Jr. R. T. Compton. Improving the performance of a slotted aloha packet radio network with an adaptive array. IEEE Transactions on Communications, 40(2):292 - 300, February 1992.

[11] V. Shankarkumar Y.B. Ko and N.H. Vaidya. Medium access control protocols using directional antennas in ad hoc networks. In IEEE INFOCOM 2000, March 2000.

[12] Chavalit Srisathapornphat Zhuochuan Huang, Chien-Chung Shen. A mac protocol based on directional antenna and busy-tone for ad hoc networks. In IEEE MILCOM 2002, 2002. 